\title{
Correction to: Aggregating neighborhood information for negative sampling for knowledge graph embedding
}

\author{
Hai Liu ${ }^{1} \cdot$ Kairong Hu${ }^{1} \cdot \mathrm{Fu}$-Lee $\mathrm{Wang}^{2} \cdot \mathrm{Tianyong} \mathrm{Hao}^{1}$
}

Published online: 25 May 2020

(C) Springer-Verlag London Ltd., part of Springer Nature 2020

\section{Correction to:}

Neural Computing and Applications https://doi.org/10.1007/s00521-020-04940-5

Unfortunately, this article has been published with incorrect title. The correct title should read "Aggregating neighborhood information for negative sampling for knowledge graph embedding".

Tianyong Hao

haoty@m.scnu.edu.cn

Hai Liu

liuhai@m.scnu.edu.cn

Kairong $\mathrm{Hu}$

2018022615@m.scnu.edu.cn

Fu-Lee Wang

pwang@ouhk.edu.hk

1 School of Computer Science, South China Normal University, Guangzhou, China

2 School of Science and Technology, The Open University of Hong Kong, Hong Kong, China 